\title{
Quantum transport in graphene Hall bars: Effects of vacancy disorder
}

\author{
M. D. Petrović* and F. M. Peeters ${ }^{\dagger}$ \\ Department of Physics, University of Antwerp, Groenenborgerlaan 171, B-2020 Antwerp, Belgium \\ (Received 29 July 2016; revised manuscript received 24 October 2016; published 9 December 2016)
}

\begin{abstract}
Using the tight-binding model, we investigate the influence of vacancy disorder on electrical transport in graphene Hall bars in the presence of quantizing magnetic fields. Disorder, induced by a random distribution of monovacancies, breaks the graphene sublattice symmetry and creates states localized on the vacancies. These states are observable in the bend resistance, as well as in the total DOS. Their energy is proportional to the square root of the magnetic field, while their localization length is proportional to the cyclotron radius. At the energies of these localized states, the electron current flows around the monovacancies and, as we show, it can follow unexpected paths depending on the particular arrangement of vacancies. We study how these localized states change with the vacancy concentration, and what are the effects of including the next-nearest-neighbor hopping term. Our results are also compared with the situation when double vacancies are present in the system. Double vacancies also induce localized states, but their energy and magnetic field dependencies are different. Their localization energy scales linearly with the magnetic field, and their localization length appears not to depend on the field strength.
\end{abstract}

DOI: 10.1103/PhysRevB.94.235413

\section{INTRODUCTION}

The discovery of graphene [1], a material with a linear lowenergy spectrum, generated new interest in the quantum Hall effects governed by relativistic particles. Unusual quantum Hall resistance plateaus were observed in graphene [2,3]. Later experiments reported new, more detailed features such as the splitting of the zeroth Landau level (LL) due to breaking of the valley and spin degeneracies $[4,5]$.

Disorder in experimentally available honeycomb graphene lattices is inevitable, whether it is structural like reconstructed and non-reconstructed vacancies, substituted carbon atoms, or it originates from charged impurities such as adatoms. Therefore, disorder in graphene is a very active area of research, both experimentally, in devising ways to characterize it [6], and theoretically in studying its influence on electron transport [7,8], with even possible applications in future spintronic devices [9]. Due to the relativistic nature of its charge carriers, disordered graphene offers a tabletop environment for the study of previously experimentally unaccessible phenomena, such as the atomic collapse reported recently in charged vacancies in graphene [10]. Vacancy disorder in the case of a zero external magnetic field was extensively studied in Refs. [11-13], where new states localized around missing carbon atoms were reported. Effects of vacancies in the quantum Hall regime were studied in Refs. [14,15], which reported on the occurrence of a zero-resistance quantum Hall plateau and breaking of the Landau level degeneracy. Graphene with on-site potential disorder was also used in Ref. [16] to test a new numerical approach to calculate the Kubo conductivities.

In this paper, we simulate the transport of electrons in a Hall bar made from a single layer of graphene. Our main goal is to study the influence of various types of vacancy disorder on the electron transport in the quantum Hall regime. We report that vacancy disorder can cause the appearance of new states

\footnotetext{
*marko.petrovic@uantwerpen.be

†francois.peeters@uantwerpen.be
}

in the Landau spectrum, which are observable in the bend resistance, as well as in the total density of states (DOS) and in the distributions of eigenenergies in a closed system (a system detached from the leads). For monovacancies, the energies of these new states scale as the square root of the magnetic field, similarly to the energies of relativistic Landau levels, but with a different scaling coefficient. The local density of states (LDOS) reveals a strong localization around the monovacancy sites, with localization length proportional to the cyclotron radius. The localization on divacancies is somewhat different: their localization energy scales linearly with the magnetic field, while their localization radius appears to be constant. We further study how the electron current flows in the presence of vacancies, and what are the effects of the next-nearest-neighbor interaction (NNN).

This paper is organized as follows: In Sec. II we describe our system and methods used to obtain our results. In order to focus on specific aspects of the problem, ranging from vacancy concentration to NNN hopping, we discuss our results (Sec. III) in several subsections (from Secs. III A to III E). All these insights are combined and summarized in the last, concluding section (Sec. IV).

\section{SYSTEM AND METHODS}

The studied system is shown in Fig. 1; it is a graphene Hall bar with zigzag edges along the horizontal leads and armchair edges along the vertical leads. The width of the vertical, armchair arms $\left(w_{v}\right)$ is chosen so that the corresponding leads are metallic, meaning that there is no gap around zero energy.

We introduce vacancy disorder in this system by randomly removing carbon atoms from the graphene lattice. Three different disorder types are studied, as shown in the right insets in Fig. 1. The first is a single-vacancy/single-sublattice disorder (SVA). Here, we randomly remove carbon atoms only from one sublattice (e.g., sublattice A). The second type is ordinary single-vacancy disorder (SV), where carbon atoms are removed without any respect to the sublattice to which they belong. The third type is a double-vacancy disorder (DV), 

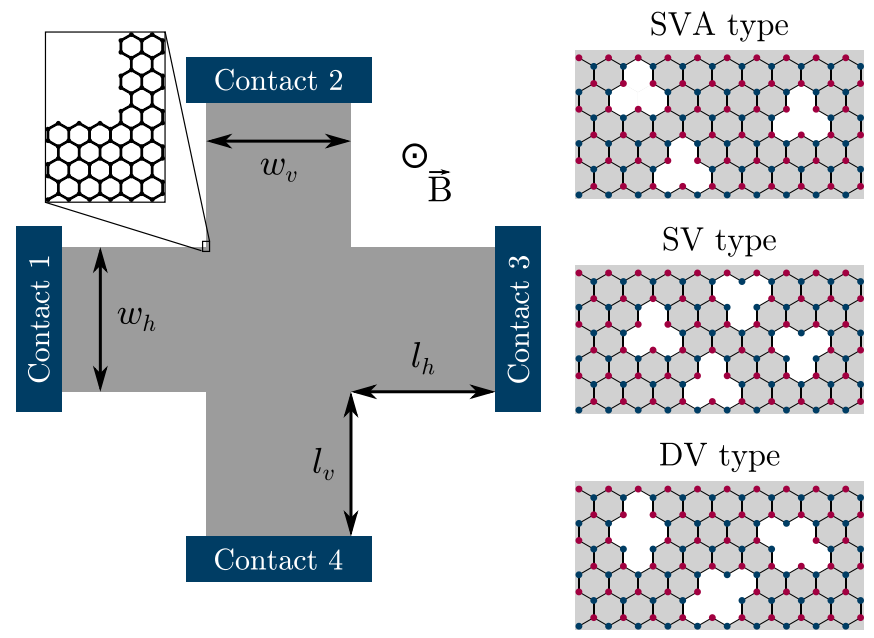

FIG. 1. Graphene Hall bar system (left) and three studied disorder types (right). Widths of the horizontal and vertical leads are set to $w_{h}=49.71 \mathrm{~nm}$ and $w_{v}=49.94 \mathrm{~nm}$, respectively, while lengths of the horizontal and vertical arms $\left(l_{v}\right.$ and $\left.l_{h}\right)$ are equal, and set to $50 \mathrm{~nm}$. Before disorder is introduced, all edges are considered to be clean, meaning that there are no dangling bonds on them.

where only pairs of neighboring atoms, each belonging to a different sublattice, are removed. It is known that single vacancies (or monovacancies) break the sublattice symmetry, while divacancies preserve it. Here we choose two types of monovacancy distributions, since one (SV) should preserve the sublattice symmetry on average, while the other (SVA) is the extreme case of sublattice symmetry breaking.

When discussing the effects of vacancy disorder, it is important to investigate the general effects introduced by disorder, and to separate them from effects that occur only for some specific disorder distributions. Therefore, we will present two types of results. In order to capture the general disorder effects, for each disorder type and concentration, we perform calculations over a sample of $N=10$ different vacancy distributions. Results for specific distributions $R_{i}$ $(i=1,2, \ldots, 10)$ are then averaged $\bar{R}=\sum_{i=1}^{N} R_{i} / N$ (we mark the averaged quantities with a bar line on top). On the other hand, in order to better understand the origin of these effects, we often analyze results for some specific distribution, or compare results of several different distributions.

For our numerical calculations we use KWANT, a software package designed to simulate electron transport in the quantum regime [17]. KWANT is based on the so-called wave function formulation of the scattering problem, a method which is mathematically equivalent to the nonequilibrium Green's function method, but according to Ref. [17] it is numerically more stable. We define graphene material in KWANT using the tight-binding model Hamiltonian

$$
\hat{\mathbf{H}}=\sum_{\langle i, j\rangle}\left(\tilde{t}_{i j} \hat{c}_{i}^{\dagger} \hat{c}_{j}+\text { H.c. }\right)+\sum_{\langle i, k\rangle}\left(\tilde{t}_{i k}^{\prime} \hat{c}_{i}^{\dagger} \hat{c}_{k}+\text { H.c. }\right),
$$

where $\hat{c}_{i}^{\dagger}\left(\hat{c}_{i}\right)$ creates (annihilates) a $p_{z}$ electron on the $i$ th carbon atom. No external electric potential is included, except that of the back gate which controls the Fermi energy. The hopping terms $\tilde{t}_{i j}=t e^{i \varphi_{i j}}$ and $\tilde{t}_{i k}^{\prime}=t^{\prime} e^{i \varphi_{i k}}$ are defined using the electron nearest-neighbor hopping energy $t=-2.7 \mathrm{eV}$, the NNN hopping term $t^{\prime}$, and the Peierls phase factor $\varphi_{i j}$ (which we discuss below). Although most of our results deal only with nearest-neighbor interaction $\left(t^{\prime}=0\right)$, in the last subsection of the next part (Sec. IIIE) we comment on the effects of a nonzero NNN term.

Defining a magnetic field in a multilead system, where some leads point in different directions, is a problem that needs to be carefully considered. Vector potential along the leads needs to be translationally invariant in order to simulate each lead as a semi-infinite system. Following this condition, we set the vector potential in horizontal leads using the Landau gauge $\vec{A}_{H}=-B y \vec{e}_{x}$, and that in vertical leads as $\vec{A}_{V}=B x \vec{e}_{y}$. To connect these two, the gauge in the main region is set to change smoothly from $\vec{A}_{H}$ to $\vec{A}_{V}$ in the upper and lower arms of the cross. This is achieved by using an additional scalar function $f(x, y)$ which rotates the vector potential $\vec{A}^{\prime}=\vec{A}+\vec{\nabla} f$ locally, without changing the orientation and strength of the magnetic field. This scalar function is defined in Ref. [18] as

$$
f(x, y)=B x y \sin ^{2} \theta+\frac{1}{4} B\left(x^{2}-y^{2}\right) \sin 2 \theta,
$$

where $\theta$ is the angle of rotation. In order to apply $f(x, y)$ only in a specific subregion of the cross, we multiply it with a smooth step function $\xi_{i}(y)=\frac{1}{2}\left\{1+\tanh \left[2\left(y-y_{0}\right) / d\right]\right\}$, which is nonzero only very close to one of the vertical leads (here the index $i$ specifies the lead number). Previous expression defines $y_{0}$ as a crossover position, where $\xi_{i}\left(y_{0}\right)=$ $\frac{1}{2}$, and $d$ as a width of the crossover region, where $\xi_{i}$ smoothly goes from 0 to 1 . For our numerical calculations, we used $d=l_{v} / 5=10 \mathrm{~nm}$. Based on this, we can define a rotation function for the second lead

$$
\begin{aligned}
F_{2}(x, y) & =f(x, y) \xi_{2}(y) \\
& =\frac{1}{2} B x y\left[1+\tanh \left(2 \frac{y-y_{u}}{d}\right)\right],
\end{aligned}
$$

and similarly for the fourth lead

$$
\begin{aligned}
F_{4}(x, y) & =f(x, y) \xi_{4}(y) \\
& =\frac{1}{2} B x y\left[1+\tanh \left(2 \frac{y_{d}-y}{d}\right)\right] .
\end{aligned}
$$

In both cases $\theta$ is set to $\pi / 2$ (since neighboring leads are perpendicular to each other), and $y_{u}=-y_{d}=\left(l_{v}+w_{h}\right) / 2$. We also define the sum of the two rotation functions as $F=$ $F_{1}+F_{2}$.

In order to check that the modified vector potential $\vec{A}^{\prime}(x, y)=\vec{A}(x, y)+\vec{\nabla} F(x, y)$ is properly defined, this function is presented in Fig. 2. The $\vec{A}_{H}$ gauge oriented in the $x$ direction in the horizontal part of the cross transforms smoothly to a $y$-oriented gauge $\vec{A}_{V}$ in the vertical part of the cross, thus confirming the correctness of $\vec{A}^{\prime}$.

Note that functions $\xi_{2}$ and $\xi_{4}$ are chosen because they are smooth, thus guaranteeing the smoothness of the vector potential. But in a tight-binding system, due to its discreteness, and the constant distance between the atoms, this is not a necessary condition. The discontinuous Heaviside step function could also be used instead. We tested this by changing the width $d$ from a value used in all our calculations $\left(d=l_{v} / 5\right)$ 


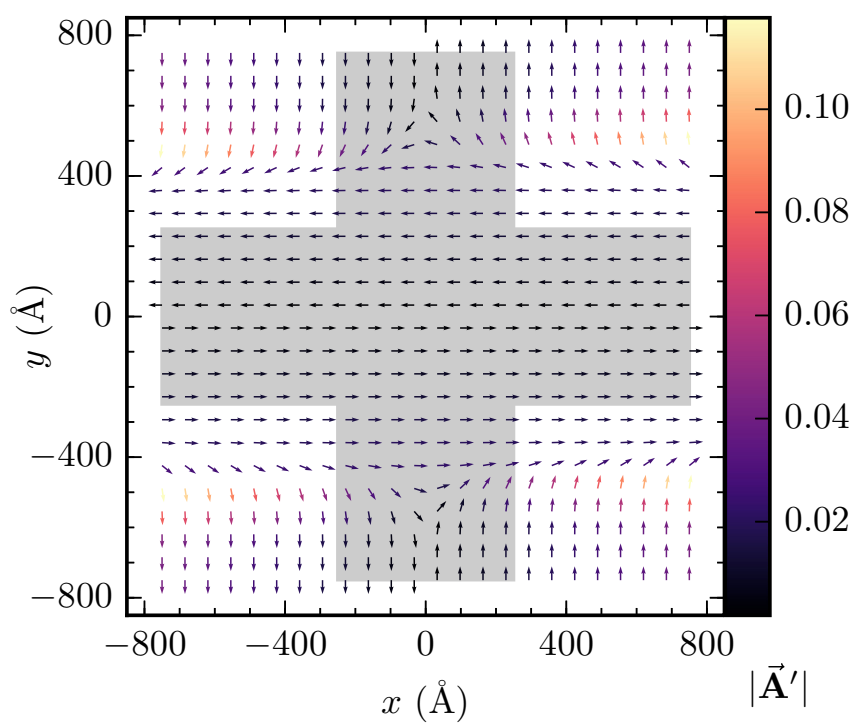

FIG. 2. Modified vector potential $\vec{A}^{\prime}(x, y)$. Arrows show the direction of $\vec{A}^{\prime}(x, y)$, and their color represents its intensity. System shape is marked by the gray area.

to values well below the carbon-carbon distance (equivalent to a discontinuous step function), with no observable changes in the final results.

The Peierls phase factor between sites $i$ and $j$ for the modified vector potential is

$$
\begin{aligned}
\varphi_{i j} & =\frac{e}{\hbar} \int_{\vec{r}_{j}}^{\vec{r}_{i}}\left(\vec{A}_{H}+\vec{\nabla} F\right) d \vec{r} \\
& =\frac{e}{\hbar} \int_{\vec{r}_{j}}^{\vec{r}_{i}} \vec{A}_{H} d \vec{r}+\frac{e}{\hbar}\left(F_{i}-F_{j}\right) \\
& =\varphi_{i j}^{L}+\Phi_{i}-\Phi_{j}
\end{aligned}
$$

where $\varphi_{i j}^{L}$ is the Peierls phase factor for the translationally invariant Landau gauge in the $x$ direction

$$
\varphi_{i j}^{L}=-\frac{e}{\hbar} B \frac{\left(y_{i}+y_{j}\right)}{2}\left(x_{i}-x_{j}\right),
$$

as is also explained in Ref. [19]. Note that $\varphi_{i j}^{L}$ does not depend on the $x$ coordinates, since differences $x_{i}-x_{j}$ are constant.

Resistances in this four-terminal device are obtained using the Landauer-Büttiker formula [20-22]

$$
R_{m n, k l}=\frac{h}{2 e^{2}}\left(T_{k m} T_{l n}-T_{k n} T_{l m}\right) / D
$$

where $R_{m n, k l}$ is a resistance measured when the current is injected from lead $m$ and collected at lead $n$, and the voltage is measured between leads $k$ and $l . T_{i j}$ is the transmission function between the corresponding leads, while parameter $D$ is defined as

$$
D=\left(\alpha_{11} \alpha_{22}-\alpha_{12} \alpha_{21}\right) S,
$$

where

$$
\begin{aligned}
& \alpha_{11}=\left(T_{21}+T_{31}+T_{41}\right)-\left(T_{14}+T_{12}\right)\left(T_{41}+T_{21}\right) / S, \\
& \alpha_{22}=\left(T_{12}+T_{32}+T_{42}\right)-\left(T_{21}+T_{23}\right)\left(T_{12}+T_{32}\right) / S, \\
& \alpha_{12}=\left(T_{12} T_{34}-T_{14} T_{32}\right) / S, \\
& \alpha_{21}=\left(T_{21} T_{43}-T_{41} T_{23}\right) / S,
\end{aligned}
$$

with

$$
S=T_{12}+T_{14}+T_{32}+T_{34}
$$

The previous resistance formula [Eq. (7)] defines six different resistances, and when used with transmission functions at a specific Fermi energy $T_{i j}\left(E_{F}\right)$ it provides resistances for the zero-temperature case. To obtain the resistances at a nonzero temperature, the previously calculated transmission functions need to be additionally convoluted in energy

$$
T_{i j}^{\prime}\left(E_{F}, T\right)=\int_{-\infty}^{\infty} T_{i j}\left(E^{\prime}\right) F_{T}\left(E^{\prime}-E_{F}\right) d E^{\prime},
$$

where the convolution function [23]

$$
F_{T}(E, T)=\frac{1}{4 k_{B} T} \operatorname{sech}^{2}\left(\frac{E-E_{F}}{2 k_{B} T}\right)
$$

is the temperature-dependent negative derivative $F_{T}(E)=$ $-\partial f / \partial E$ of the Fermi-Dirac distribution

$$
f(E)=\frac{1}{\exp \left[\left(E-E_{F}\right) / k_{B} T\right]+1} .
$$

Since vacancy disorder introduces a considerable amount of noise in all calculated quantities, in some cases we perform temperature smoothing by setting $T=16 \mathrm{~K}$; the temperature is considered to be zero otherwise. In the case of the averaged results, the temperature smoothing is always performed before the averaging.

\section{RESULTS}

\section{A. Effects of different disorder types}

Here we discuss the general transport effects of the three disorder types, observable in the Hall $\left(R_{H}=R_{13,42}\right)$ and the bend $\left(R_{B}=R_{12,43}\right)$ resistances. Note that actual Hall measurements are usually performed on devices with six or more terminals, with current and voltage probes usually set on different terminals. That is why we focus here on the bend resistance $R_{B}$, and not on $R_{13,13}$, since $R_{B}$ should be closer to experimentally measured $R_{x x}$.

It is widely known [24] that the Hall resistance in graphene exhibits quantized plateaus

$$
R_{H}=\left(\frac{h}{2 e^{2}}\right) \frac{1}{1+2 n}, \quad n=0, \pm 1, \ldots,
$$

between the energies of the Landau levels

$$
E_{n}=\operatorname{sgn}(n) \sqrt{2 e B v_{F}^{2} \hbar|n|},
$$

where $n$ is the Landau level number, and $v_{F}$ is the Fermi velocity $\left(v_{F}=3|t| a / 2 \hbar \approx 10^{6} \mathrm{~m} / \mathrm{s}\right.$, with parameter $a=1.42 \AA$ being the carbon-carbon distance). At the steps in the Hall resistance, the longitudinal resistance exhibits peaks. Beside 


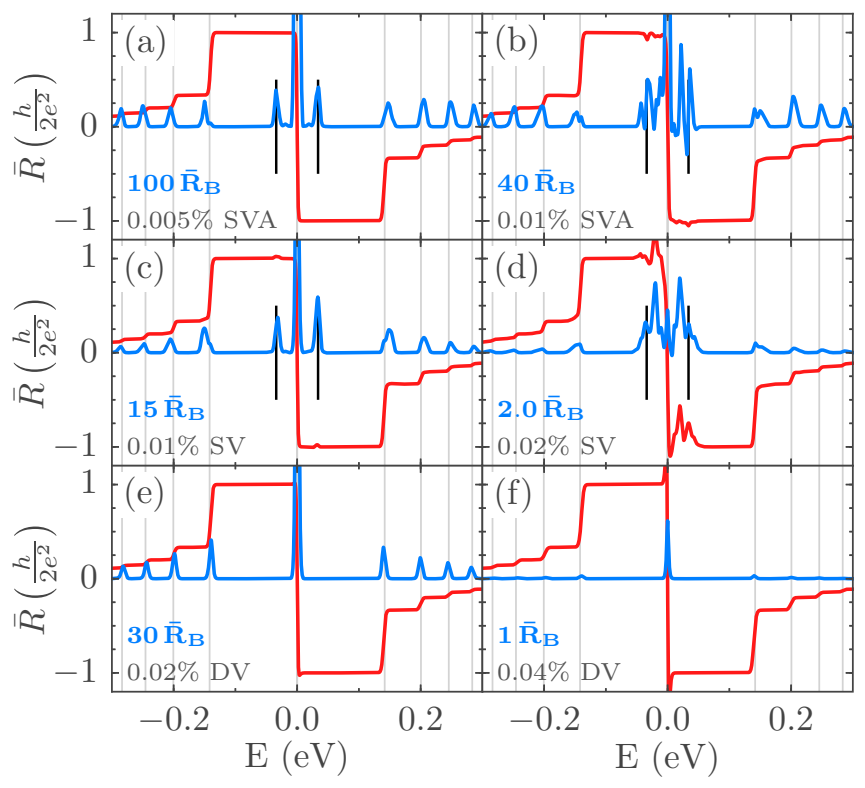

FIG. 3. Average Hall ( $\bar{R}_{H}=\bar{R}_{13,42}$; red curves) and bend $\left(\bar{R}_{B}=\right.$ $\bar{R}_{12,43}$; blue curves) resistances for various types and concentrations of vacancy disorder. Disorder type and concentration are shown in every subplot, in the lower-left corner. Results for the bend resistance depend strongly on the vacancy concentration; therefore we scale $\bar{R}_{B}$ by multiplying it with a scaling coefficient, in order to present all results in the same range. A scaling coefficient is presented in every subplot, above the vacancy concentration. Thin gray vertical lines mark the energy of Landau levels given by Eq. (15) for $B=20 \mathrm{~T}$, while black vertical lines mark the position of the new peaks at $E= \pm 33.9 \mathrm{meV}$. The temperature is equal to $16 \mathrm{~K}$.

these expected features, our results for a disordered system (presented in Fig. 3) show some additional features.

Monovacancy disorders (SV and SVA) induce two new peaks in the bend resistance, around $E= \pm 33.9 \mathrm{meV}$. These peaks do not agree with the analytic formula for Landau levels given by Eq. (15). In contrast, peaks induced by double vacancies (DV) appear to agree with Eq. (15) (i.e., they correspond to the expected Landau levels, broadened by temperature and vacancy scattering). Each row in Fig. 3 presents data for one type of vacancy disorder, for two different concentrations. For each vacancy type, the increase in vacancy concentration leads to an increase in $R_{B}$, which can be seen in a decreasing scaling coefficient (given in the insets of the figure). The higher the vacancy concentration, the larger the bend resistance, and consequently the smaller the scaling coefficient. For higher concentrations [Figs. 3(b) and 3(d)], two peaks in $\bar{R}_{B}$ are not well defined, and $\bar{R}_{H}$ also slightly deviates from the expected Hall plateaus. Although we discuss the effects of a vacancy concentration further below, it is important to state that new peaks in $\bar{R}_{B}$ occur only in a certain range of concentrations, and that above some critical concentration, these peaks broaden and merge. This critical concentration depends on the ratio of the average vacancyvacancy distance and the magnetic length. It also depends on the type of monovacancy disorder, since for SVA disorder, the two peaks disappear for smaller concentrations $[0.01 \%$ in Fig. 3(b)] as compared to SV disorder [0.02\% in Fig. 3(d)].
Another interesting feature is the negative bend resistance in Fig. 3(b). As explained in Ref. [21] [page 321, paragraph below Eq. (13) in that reference] and in Ref. [22] (Sec. 3.4.4.2 in that reference), the Büttiker formula for a four-terminal device can produce negative nonlocal resistances. This is usually the case when the second term in the numerator of Eq. (7) is larger than the first term. We obtain negative $R_{B}$ peaks for almost all concentrations, but for low concentrations they do not appear often (because the scattering is weak), and are not very pronounced (they usually disappear after temperature smearing). In general, if the number of vacancies exceeds the critical value, vacancy scattering becomes too strong, such that no general features exist in the low-energy region. The bend resistance then strongly depends on a particular vacancy distribution.

Another characteristic of the averaged resistance $\bar{R}_{B}$ is that it is fairly symmetric with respect to electrons and holes, whereas results for individual distributions (used to calculate $\bar{R}_{B}$ ) are not. This means that in general, a random monovacancy distribution induces two new peaks in the bend resistance, but the actual relative height of those two peaks depends on a particular arrangement of vacancies. For some distributions there is only one peak in $R_{B}$, at positive or negative energy, and for some distributions there are no peaks at all (a question which we address in Sec. III D). This asymmetry between electrons and holes is expected, since exchanging electrons for holes is equivalent to flipping the magnetic field, which in turn is equivalent to keeping the field fixed and flipping the system around the $z$ axis. A clean system is symmetric with respect to this transformation, but a disordered system is not. After the flip, the incoming electrons see a different arrangement of vacancies. A vacancy distribution can be constructed to be symmetric with this flip transformation, in which case all results would also be electron-hole symmetric. This asymmetry between electrons and holes occurs only for a fixed field orientation $\left[R_{B}(E, B) \neq R_{B}(-E, B)\right]$, and should not be confused with the case when both magnetic field and Fermi energy change sign. Results for electrons and holes are then symmetric: $R_{B}(E, B)=R_{B}(-E,-B)$.

Results for the averaged total density of states (DOS) and distributions of eigenlevels in a closed system, presented in Fig. 4, are obtained for the same set of vacancy distributions as in Fig. 3, and they exhibit similar effects to those seen in the bend resistance in Fig. 3. Here, as in Fig. 3, monovacancy distributions induce two new broad peaks in the total DOS (around $E= \pm 33.9 \mathrm{meV}$; marked with red lines in Fig. 4), while double-vacancy distributions appear only to broaden the DOS around the expected Landau levels. We show below that the energy of these broadened peaks $(E= \pm 33.9 \mathrm{meV})$ corresponds to an energy of a monovacancy localized state. Similar behavior is seen in distributions of eigenlevels in a closed system (a Hall bar detached from the leads). Usually, the eigenlevels of a closed system in a high magnetic field tend to cluster around the energies of the Landau levels. Here we plot histograms (orange areas in Fig. 4) showing how many eigenlevels occupy a narrow energy range around each energy, and these plots also show two distributions around $E= \pm 33.9 \mathrm{meV}$.

According to Refs. [14,25], divacancies in graphene should also induce new states in the Landau spectrum. Our results 


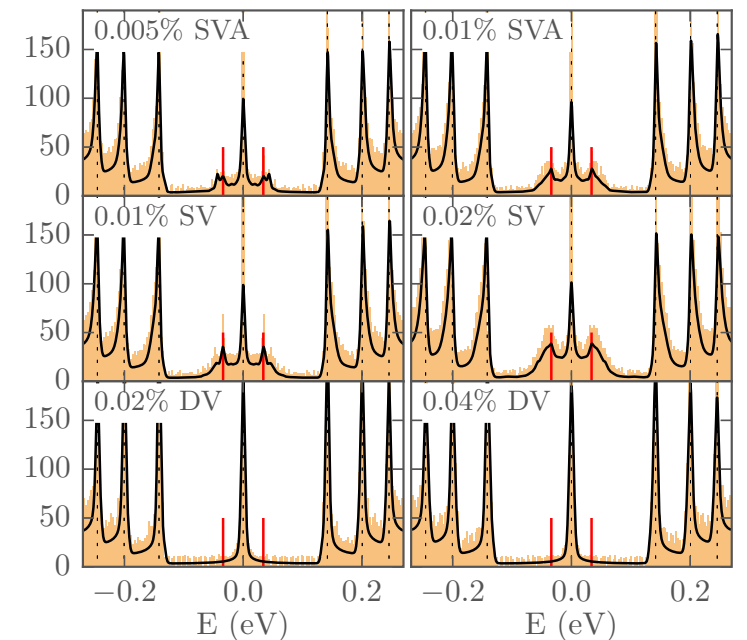

FIG. 4. Averaged DOS (black curves; arbitrary units) and distributions of eigenenergies in a closed system (orange histograms in the background). Red lines mark the positions of the two new peaks at $E= \pm 33.9 \mathrm{meV}$, while dotted lines mark the energies of Landau levels. Similarly to the resistances in Fig. 3, for every vacancy type and concentration, DOS is first smoothed and then averaged over $N=10$ different vacancy samples. In the case of the eigenenergies, results for positive energies for $N=10$ distributions are summed without smoothing or averaging, and mirrored around $E=0$ axis. We used the same sets of vacancy distributions as in Fig. 3. Magnetic field is $B=20 \mathrm{~T}$, and $T=16 \mathrm{~K}$.

for DV distributions appear to contradict those of these two references. However, a higher resolution DOS plot around the zeroth Landau level [shown in Fig. 5(a)] for one particular DV distribution reveals additional DOS peaks. These peaks are positioned only a few meV away from the LLs, and that is why they were not very distinguishable from the LLs in the previous results. This suggests that additional peaks coming from the divacancies would be harder to observe experimentally, since they would be usually smeared by temperature.

Previous experiments on graphene in high magnetic fields [5] reported splitting of the zero Landau level, which was attributed to the breaking of the sublattice symmetry. One of the possible explanations of the new DOS peaks is that they correspond to the occurrence of new states, localized in areas close to the vacancies. The unsplit zeroth Landau level is still present in the DOS of the whole device (for all disorder types), since it originates from the local density of states (LDOS) in areas which are vacancy free. This connection between the new DOS peaks and the vacancy localized states becomes apparent if we look at the LDOS at one of the two peak energies. A LDOS at one of the two new peaks, for one particular SVA distribution, is shown in Fig. 5(b). The LDOS is highly localized around the vacancies (marked with green circles). A zoom in Fig. 5(c) shows states localized mostly on one sublattice, which could be connected with the breaking of sublattice symmetry. These states that are localized around single vacancies are the origin of the two new peaks in $R_{B}$ and DOS. Similarly, in Figs. 5(d) and 5(e) we show the LDOS for one particular DV distribution, at the energy of one of the new peaks [marked with red line in Fig. 5(a)]. Divacancies also induce localized states, but these states are localized
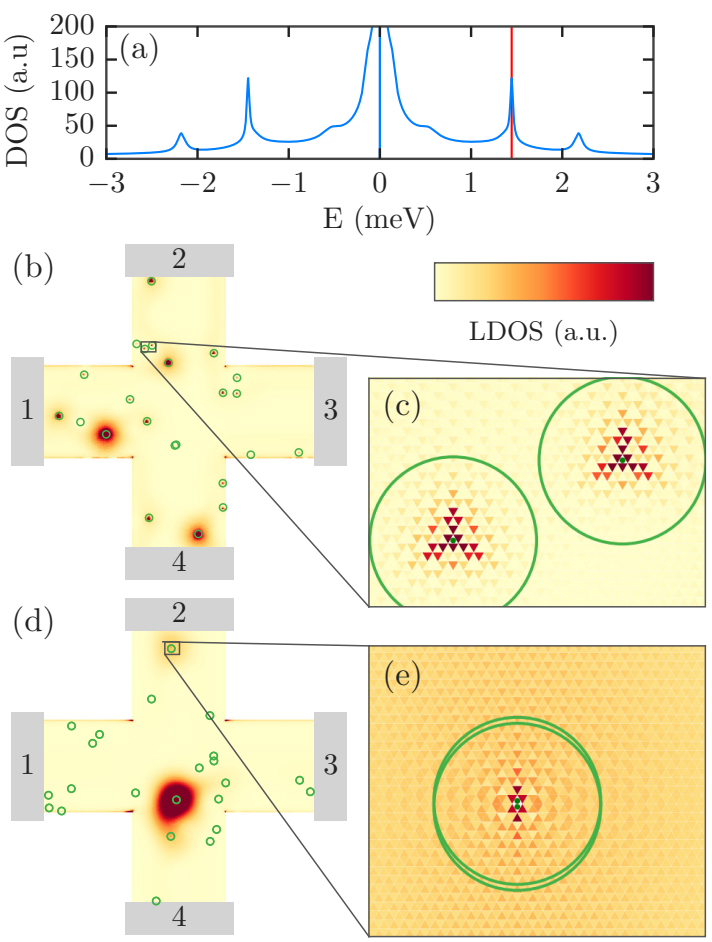

FIG. 5. (a) DOS for a single DV distribution $(n=0.01 \%, B=$ $14 \mathrm{~T})$. (b) LDOS for a single SVA distribution $(n=0.005 \%, B=20$ $\mathrm{T})$ at the vacancy localization energy $(E=33.9 \mathrm{meV})$. (d) LDOS for a single DV distribution $(n=0.01 \%, B=14 \mathrm{~T})$ at the localization energy [ $E=1.45 \mathrm{meV}$; marked with red vertical line in (a)]. (c) and (e) Zoom around particular vacancies in (b) and (d). Vacancies are marked with green circles and green dots in the center. Temperature is set to $T=0 \mathrm{~K}$.

equally on both sublattices, since divacancies do not break the sublattice symmetry. For other energies (when there is no localization), divacancies act similarly to graphene structural armchair edges; namely, the LDOS spreads in areas between them, as if they repel it. Similar behavior was observed in Ref. [14].

\section{B. Changing vacancy concentration}

As stated previously, all these results depend strongly on the vacancy concentration. To illustrate this, we present in Fig. 6 how the DOS and LDOS change with increasing number of vacancies, belonging to a SV disorder type. Here, we show results for specific vacancy distributions without any temperature smoothing or averaging. For low concentrations [Figs. 6(a) and 6(e)] the DOS shows two well-defined peaks at $\pm 33.9 \mathrm{meV}$, which correspond to one state, localized around one monovacancy. Other vacancies in Fig. 6(a) are very close to the system edges, and localization on them is very weak. These results explain why the smoothed and averaged $R_{B}$ and DOS exhibit strong peaks around $\pm 33.9 \mathrm{meV}$, because this corresponds to the energy of a state localized around one isolated monovacancy. The localization happens at this specific energy only if a vacancy is in the bulk and sufficiently away from the system edges, but also far from the other vacancies, which is satisfied only for low concentrations. 

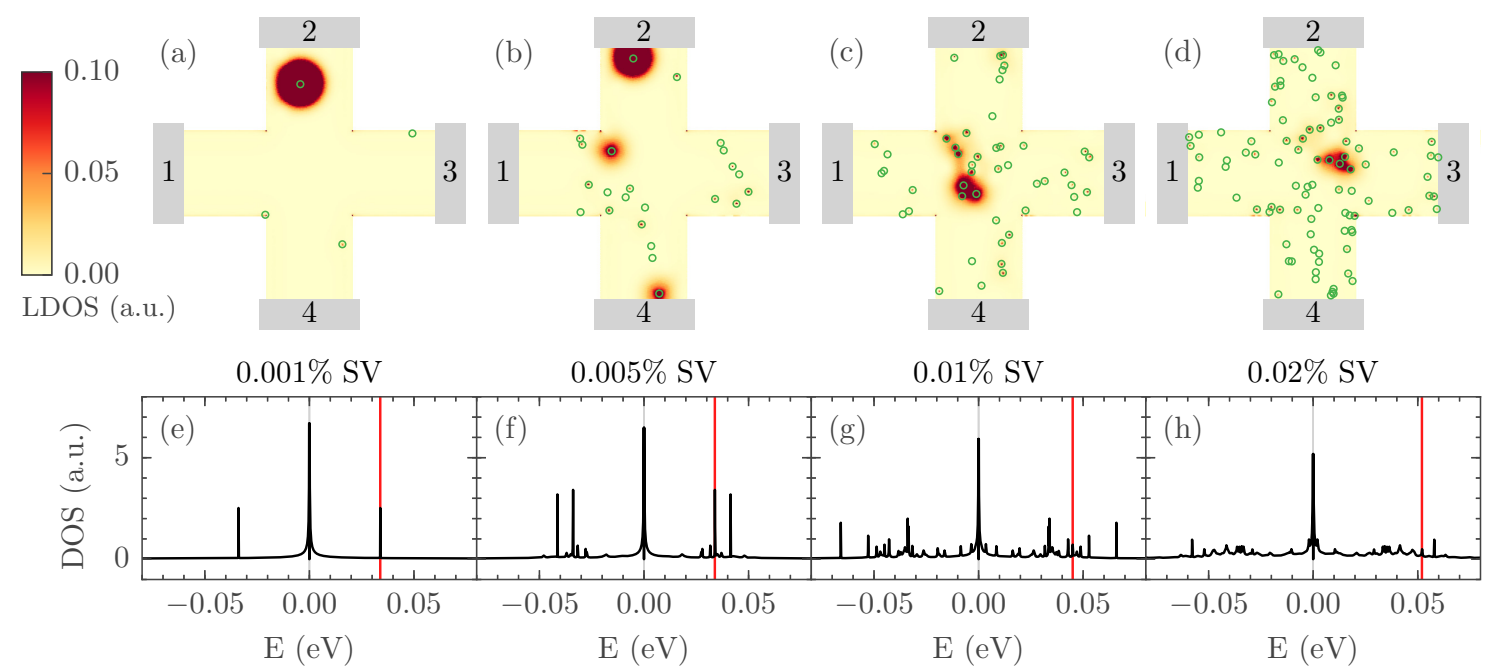

FIG. 6. (a)-(d) LDOS for SV disorder type, for different vacancy concentrations: $0.001 \%, 0.005 \%, 0.01 \%$, and $0.02 \%$, and at different energies: $33.9 \mathrm{meV}, 33.9 \mathrm{meV}, 45 \mathrm{meV}$, and $52 \mathrm{meV}$, respectively. Vacancy positions are marked with green circles. (e)-(h) DOS for SV disorder type, for the corresponding concentrations. Energies at which we calculated LDOS in (a)-(d) are marked with red vertical lines in (e)-(h). Magnetic field is $B=20 \mathrm{~T}$, and $T=0 \mathrm{~K}$.

With increasing concentration, the average distance between the vacancies decreases, and vacancies start to "see" each other, meaning that they start to influence the formation of localized states on their neighbors. This is demonstrated in Figs. 6(b) and 6(f), where several peaks appear in the total DOS. However, the peak at the monovacancy localization energy $( \pm 33.9 \mathrm{meV})$ is still well defined. This is because there is still one well isolated monovacancy in the upper arm of the cross [see Fig. 6(b)]. For even higher concentrations, there are no longer well isolated vacancies, and therefore there is no well defined localization energy. Instead, the monovacancies start to form something which resembles bond states. In a vague analogy with atoms and molecules, these bond states correspond to groups of vacancies which are sufficiently close to each other, so that localization occurs over the whole group, and not on separated, individual vacancies. This bonding, shown in Figs. 6(c) and 6(d), is responsible for spreading of the localization energy, and consequently for broadening of the new peaks in $R_{B}$ and DOS.

\section{Changing the magnetic field}

In this part, we investigate how these vacancy localized states behave when we change the magnetic field $B$. In Figs. 7(a) and 7(b) we show that the localization energy for monovacancies scales with the square root of the magnetic field $E \sim \pm \sqrt{B}$, similarly to the relativistic Landau levels. The blue curves in Figs. 7(a) and 7(b) show the parabolic function $B=\alpha E^{2}$, where parameter $\alpha=17500 \mathrm{~T} /(\mathrm{eV})^{2}$ is set to fit the peak positions. This dependence can also be expressed as $E= \pm \sqrt{\gamma 2 e v_{F}^{2} \hbar B}$, where $\gamma \approx 0.057$. It is important to note that Fig. 7(a) presents results for the same vacancy distribution as in Fig. 6(a), with only one monovacancy capable of sustaining the localized states. The DOS in this case exhibits two narrow peaks at positive and negative localization energy. For weak fields $(B<5 \mathrm{~T})$ these peaks are almost unobservable, whereas for stronger fields they become better and better defined in energy. Beside these two localization peaks, Fig. 7(a) shows some additional peaks for $B=0 \mathrm{~T}$ (e.g., two peaks at approximately $\pm 35 \mathrm{meV}$ ). According to Pereira et al. [11], localization of electrons on vacancies also occurs for $B=0 \mathrm{~T}$, but localization energy is then equal to zero, and therefore these extra peaks should not be connected with the localized states. Indeed, a closer study reveals that these peaks originate from new modes opening in the leads, and can be predicted by calculating the lead minimal subband energies.

For larger concentration of monovacancies [Fig. 7(b)], the localization energy is not well defined, and the two narrow

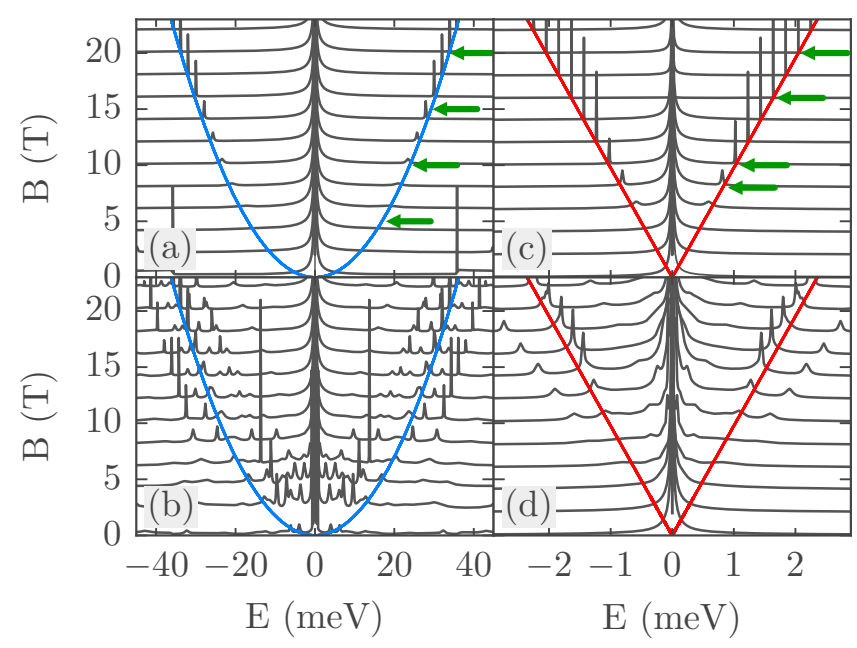

FIG. 7. DOS for different values of magnetic field and different disorder distributions: (a) $n=0.001 \% \mathrm{SV}$ distribution, (b) $n=$ $0.005 \% \mathrm{SV}$ distribution, (c) single divacancy located at the center of the system, (d) $n=0.01 \%$ DV distribution. The two distributions of monovacancies are the same as those used in Figs. 6(a) and 6(b), respectively. In all four cases $T=0 \mathrm{~K}$. The green arrows mark $(E, B)$ points at which we study LDOS in Figs. 8, 9, and 10. 
DOS peaks from Fig. 7(a) split into two distributions of peaks. As we explained in the previous subsection, this is mainly due to a decrease of the average vacancy-vacancy distance, and is thus due to an increase of the interference between vacancies, resulting in the formation of bond localized states. Since localization radius around a monovacancy is inversely proportional to the square root of the magnetic field (as we show below), the field strength determines how far a single vacancy actually "sees" its surroundings; i.e., it determines the bond length of previously described bond states. Because this length changes with magnetic field, various groups of vacancies bond together at different field strengths, and the two distributions of DOS peaks in Fig. 7(b) evolve quite unpredictably with $B$. However, the average energies of the two distributions still tend to follow the parabolic $B$ dependence, as is apparent from the graph. Thus for extremely large fields, the localization would be so strong that the bond length will go below the average vacancy-vacancy distance, and the vacancies would no longer "see" each other. All these separate DOS peaks would then converge to a single energy, equal to that of an isolated monovacancy.

Scaling of the localization energy with magnetic field is different for divacancies. As Figs. 7(c) and 7(d) show, the localization energy for divacancies scales linearly with the magnetic field. The red lines in these two figures mark the linear dependence $B=\beta E$, where $\beta \approx 9700 \mathrm{~T} / \mathrm{eV}$. Contrary to monovacancies where the bond states evolve rather unpredictably with magnetic field, the bond states of divacancies evolve very predictably with the field. The two DOS peaks at positive energies and the two peaks at negative energies in Fig. 7(d) move proportionally to the magnetic field. There are no additional peaks which would correspond to different bonding of divacancies. These results suggest that bonding of divacancies is weaker, when compared to monovacancies. One of the possible reasons for this weaker bonding might be the constant localization length for divacancies, which we discuss below.

Reference [25] also studied the $E(B)$ dependence of the new (localized) states, and for both mono- and divacancies found it to be neither linear, nor parabolic. However, the lowest field considered in that reference (beside $B=0 \mathrm{~T}$ ) was around $300 \mathrm{~T}$; therefore our results can be understood as a low field limit of those presented in Ref. [25].

As we stated previously, the localization radius for monovacancies $r_{L}$ is inversely proportional to the square root of the magnetic field. It is also proportional to the cyclotron radius $r_{L} \sim R_{c}=E /\left(e v_{F} B\right)$, and since $E \sim \sqrt{B}$, then $r_{L} \sim 1 / \sqrt{B}$. To demonstrate this, in Fig. 8 we follow how the LDOS around an isolated monovacancy evolves as we increase the magnetic field. In other words, we follow the localized state along the $\alpha E^{2}$ parabola in Fig. 7(a). A first look at Fig. 8 suggests that localization radius is not proportional to the cyclotron radius $R_{c}$. While $R_{c}$ decreases with rising magnetic field, the localization radius appears to increase, and the LDOS forms intricate flower-like patterns. The answer to this contradiction lies in the lower-right insets in Fig. 8, which show the total DOS around the localization energy. For stronger fields, the localized state is better defined in energy, and therefore the total DOS is larger. In order to properly compare these four

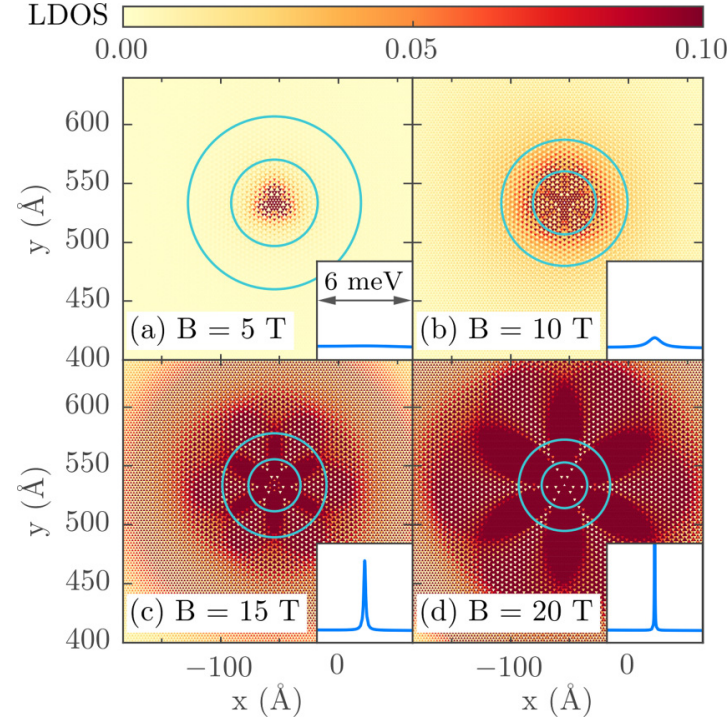

FIG. 8. The evolution of LDOS around a single SV vacancy for $(E, B)$ values lying on the parabola in Fig. 7(a) (marked by the green arrows). The magnetic field strengths are $5 \mathrm{~T}, 10 \mathrm{~T}, 15 \mathrm{~T}$, and $20 \mathrm{~T}$, and the corresponding energies and cyclotron radii are (a) $E=16 \mathrm{meV}, R_{c}=36.7 \AA$; (b) $E=23.4 \mathrm{meV}, R_{c}=26.8 \AA$; (c) $E=29 \mathrm{meV}, R_{c}=22.1 \AA$; and (d) $E=33.9 \mathrm{meV}, R_{c}=19.4 \AA$. The two circles with radii $R_{c}$ and $2 R_{c}$ in each inset are centered at the vacancy site. Insets in the lower-right corners show the total DOS in a $6 \mathrm{meV}$ energy range around the localization energy.

cases, we need to normalize the LDOS in each subplot. This is done in Fig. 9, where each LDOS distribution is divided by its maximal value. Now, with these normalized results, the localization radius scales proportionally to the cyclotron

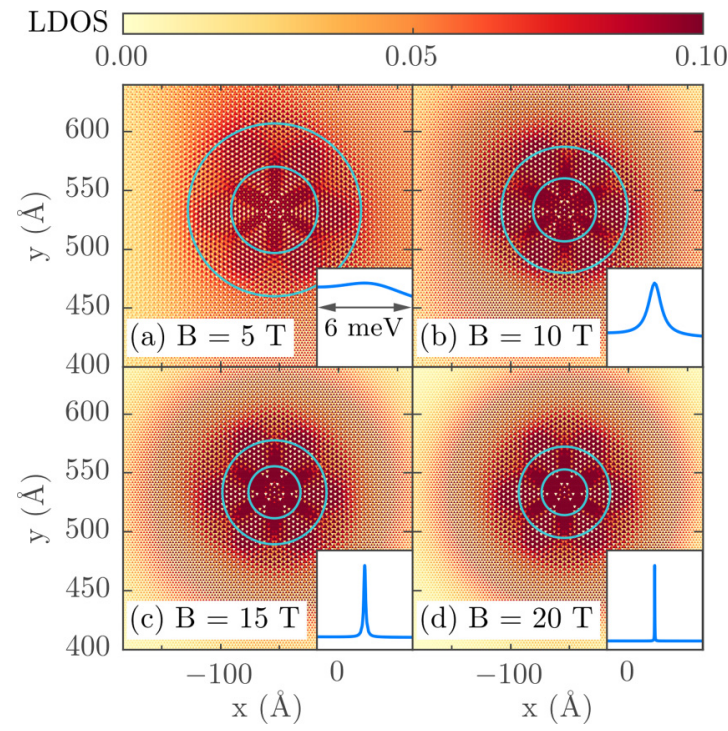

FIG. 9. Same as Fig. 8 but now showing normalized LDOS, where LDOS in each subplot is divided by a maximum LDOS value for that subplot. The lower-right insets (showing the total DOS around the energy of a localized state) are also scaled, so that the DOS peak maximum is equal to 1 . 


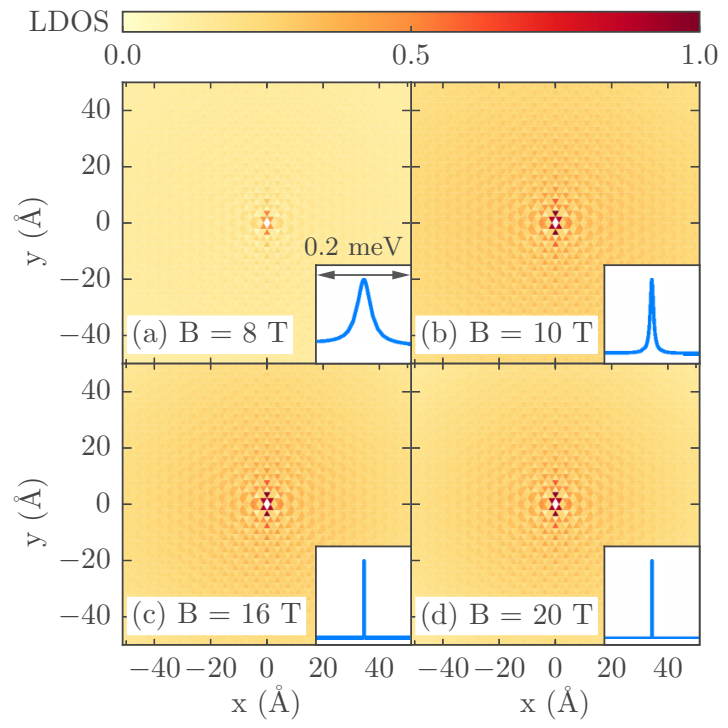

FIG. 10. Same as Fig. 9 but now showing normalized LDOS and DOS for a single divacancy located at the center of the system. The $(E, B)$ points at which we calculated LDOS and DOS are marked with green arrows in Fig. 7(c).

radius $R_{c}$ as is intuitively expected. The scaled results also point to another interesting feature. We stated earlier that the LDOS around a monovacancy is localized mostly on one sublattice, opposite to that of the vacancy. However, the scaled results show that at the localization energy, the LDOS around a monovacancy spreads over both sublattices. Nonzero LDOS on the vacancy sublattice is located mostly in the symmetric, flower-like area. Outside of this area, states are still localized only on one sublattice. LDOS is also $C_{3 v}$ symmetric, which can be connected with the underlying $C_{3 v}$ (structural) lattice symmetry.

A similar LDOS comparison, but for an isolated divacancy, is presented in Fig. 10. Contrary to monovacancies, a divacancy localization length does not change significantly with magnetic field. This can be understood just based on the linear $E(B)$ dependence of the divacancy localization energy. If we assume that localization length is still proportional to the cyclotron radius, then since $E=B / \beta$, it follows that $r_{L} \sim$ $R_{c}=E /\left(e v_{F} B\right)=1 /\left(\beta e v_{F}\right)$. The LDOS around a divacancy is $C_{2 v}$ symmetric, which could be also connected with the underlying lattice symmetry. Contrary to monovacancies, divacancies preserve the sublattice symmetry, and this is the origin of the different behavior of these two disorder types.

\section{Decomposition of $\boldsymbol{R}_{B}$ and the current density}

When discussing results for the averaged bend resistances $\bar{R}_{B}$ in Sec. III A, we mentioned that although the averaged results appear to be symmetric for electrons and holes, the results for individual distributions are not, and for some distributions there are no new peaks in $R_{B}$. In this subsection we study why this is the case. We compare bend resistances for two specific monovacancy distributions: one for which there are new peaks in $R_{B}$, and one for which there are not. In order to understand how these peaks come into existence from the different transmission terms in the $S$ matrix, we decompose

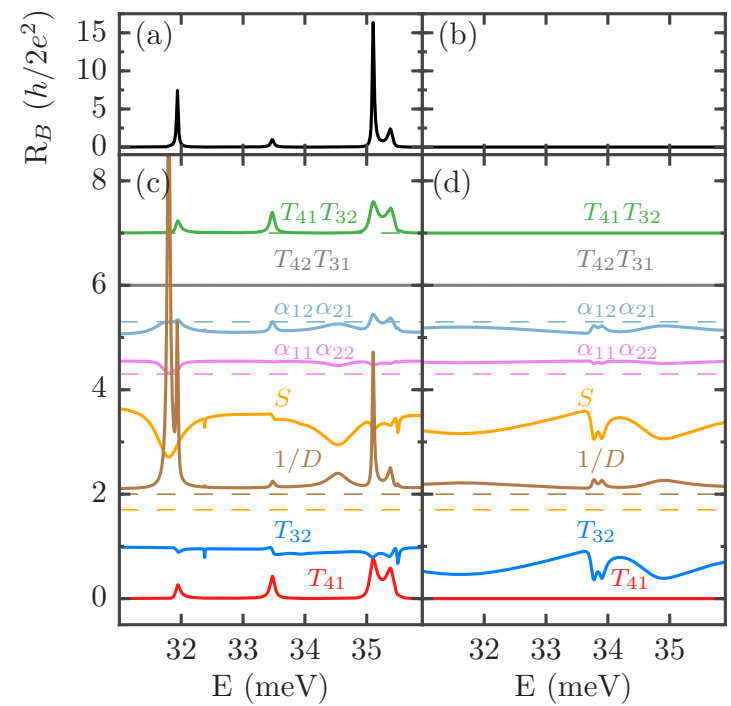

FIG. 11. Decomposition of the bend resistance $R_{B}=$ $\left(T_{41} T_{32}-T_{42} T_{31}\right) / D$, where $D=\left(\alpha_{11} \alpha_{22}-\alpha_{12} \alpha_{21}\right) S$. Results for two SV distributions $(n=0.01 \%)$ in (a) and (b) are decomposed in (c) and (d), respectively. Most of the terms in (c) and (d) are vertically displaced, with dashed lines marking the corresponding positions of the zero axes. Magnetic field is $B=20 \mathrm{~T}$, and $T=0 \mathrm{~K}$.

$R_{B}$ on its constituent parts, according to the Landauer-Büttiker (LB) formula [20]. Results are presented in Fig. 11. Here we focus only on a narrow energy range where these new peaks in $R_{B}$ appear. Analysis of the main LB terms in Fig. 11(c) reveals that only one term $\left(T_{41} T_{32}\right.$; green curve $)$ is responsible for the appearance of the $R_{B}$ peaks. The other term in the numerator ( $T_{42} T_{31}$; gray line) is always equal to zero. A further decomposition of the first term $\left(T_{41} T_{32}\right.$; green curve) shows that one transmission function ( $T_{32}$; blue curve) is very close to unity, and that only $T_{41}$ (red curve) dictates where the new $R_{B}$ peaks appear. Only when this transmission $\left(T_{41}\right)$ is nonzero, we have peaks in $R_{B}$. Therefore, to a first approximation, we can say that $R_{B}$ is proportional to modulated $T_{41}$. One might argue that $T_{32}$ is also important, but since $B$ is perpendicular, $T_{32}$ will always be close to unity in this energy range, because of the edge states that go from the second to the third lead. This $R_{B}-T_{41}$ connection is also confirmed in Figs. 11(b) and 11(d), where both main LB terms in the numerator are equal to zero, as well as $T_{41}$, and thus $R_{B}$ is also equal to zero.

The only way to understand why for some vacancy distributions the particular $T_{41}$ transmission is equal to zero, and for some it is not, is to investigate how electron current flows in the presence of vacancy disorder. This is presented in Fig. 12, for the same two SV distributions as those used in Fig. 11. In a clean system without vacancies, and with a perpendicular magnetic field, all current from the first lead would go to the second lead because of the current carrying edge states. This is mostly what we see in both cases [Figs. 12(a) and 12(b)] where the $T_{21}$ term is the most dominant when compared with the other transmission functions. This is also visible in Fig. 12(c), where most of the current from the first lead travels to the second lead along the edges. The two vacancy distributions differ in the way they scatter this edge current from the first to the second lead. The first distribution [Fig. 12(a)] is 

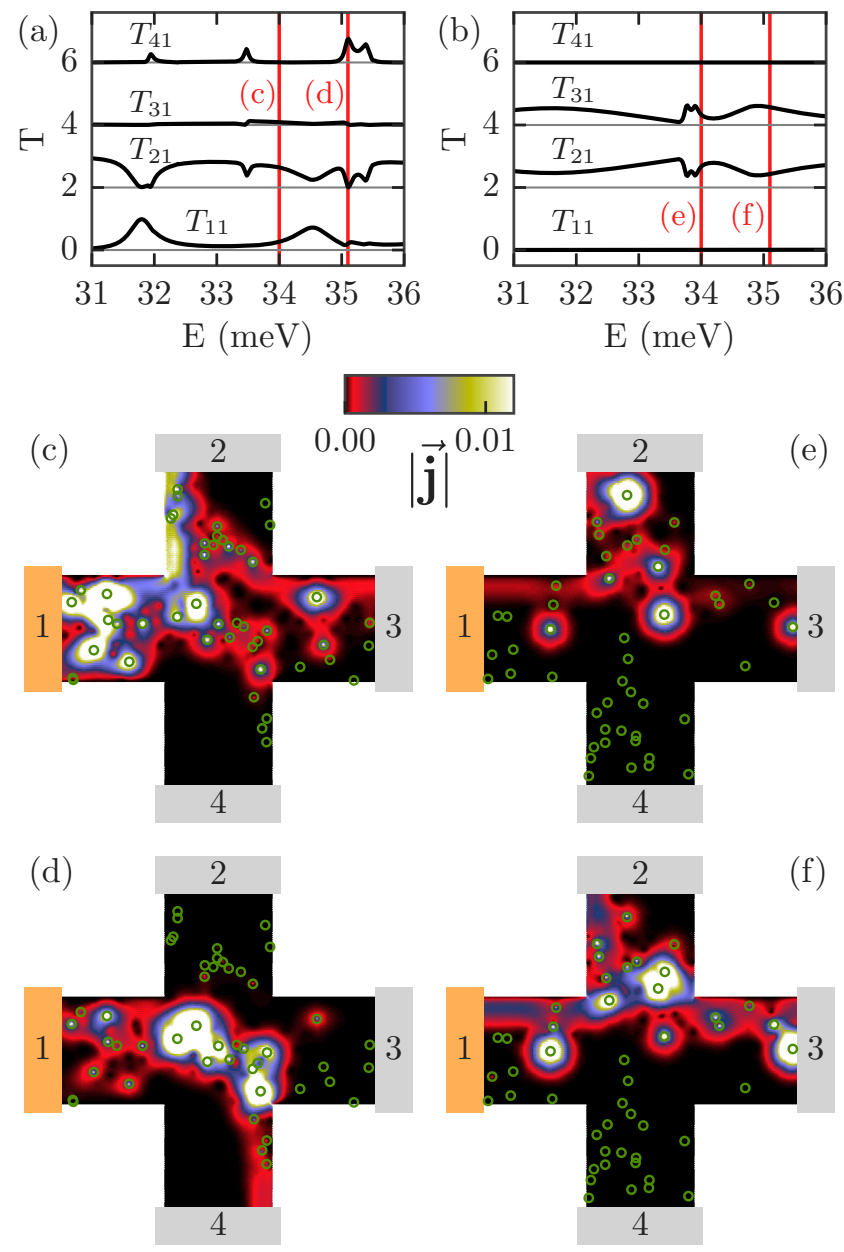

FIG. 12. (a) and (b) Electron transmission functions from the first lead, for two specific disorder distributions [the same two distributions as used in Figs. 11(a) and 11(b), respectively]. The transmission functions are vertically displaced by $\Delta T=2$ for clarity. (c)-(f) Current densities at energies marked in (a) and (b) by vertical red lines. Magnetic field is $B=20 \mathrm{~T}$, and temperature is $T=0 \mathrm{~K}$.

causing more backscattering $\left(T_{11}\right)$, and scattering to the fourth lead $\left(T_{41}\right)$, whereas the second distribution is causing more scattering to the third lead $\left[T_{31}\right.$ in Fig. 12(b)]. Where this edge current is diverted depends mostly on a particular arrangement of vacancies, since current flow is pinned by the vacancies. For example $T_{41}$, and consequently $R_{B}$, exhibits narrow peaks because of a particular arrangement of vacancies in the central part of the cross. As shown in Fig. 12(d), the current starts to flow around these vacancies, and it is diverted to the fourth lead. In a similar way, the current flow in Figs. 12(e) and 12(f) is also pinned by the vacancies, and diverted to the third lead.

Reference [23] (and particularly chapter IV in this reference) gives a valuable explanation of the quantum Hall effect in terms of the electron propagation along the sample edges. According to this reference, the rise of longitudinal resistance (for Fermi energies coinciding with the Landau levels) occurs due to the existence of states in the interior of the sample. These bulk states connect the otherwise separated edges channels, and give rise to their backscattering, and this backscattering manifests in a nonzero longitudinal resistance. The existence of vacancy localized states in our system, with energies in between the Landau levels, leads to the expected LL broadening. Additionally, these vacancy states can provide a narrow pathway between the channels propagating along the opposite edges of the system. In our particular setup, the nonzero $T_{41}$ term is due to a backscattering between a channel going from the 1 st to the 2 nd lead $(1 \rightarrow 2)$, and the one going from the 3 rd to the 4 th lead $(3 \rightarrow 4)$. This edge state scattering is responsible for the nonzero bend resistance.

Similar analysis can also explain the asymmetry between the resistance results for electrons and holes (when the field direction is fixed). In a clean sample with no vacancies, $T_{21}=1$ and $T_{41}=0$ for electrons, while $T_{21}=0$ and $T_{41}=1$ for holes. Also $T_{32}=1$ for electrons, while $T_{32}=0$ for holes. We already showed that the first term in the Büttiker formula [term $T_{41} T_{32}$ in Eq. (7)] determines the bend resistance. For electrons this term depends mostly on $T_{41}$, since $T_{32}=1$. For holes, on the other hand, it depends on $T_{32}$, since $T_{41}=1$. Because $T_{32}(-E, B) \neq T_{41}(E, B)$, the bend resistance in a disordered system is not the same for electrons and holes. The bend resistance becomes equal only if we additionally change the magnetic field direction (from $\vec{B}$ to $-\vec{B}$ ) when we switch from electrons to holes.

In summary, although the two new peaks in $R_{B}$ should in general appear at the vacancy localization energy, they are very sensitive to a particular distribution of vacancies. The vacancies significantly disrupt and divert the current flow. However, if not in $R_{B}$, this current guiding will probably manifest itself in measurements of some other nonlocal resistance.

\section{E. NNN interaction}

In this section we study the effects of a nonzero hopping between the second-nearest neighbors $\left(t^{\prime} \neq 0\right)$. Figure 13 shows the averaged results for the SV disorder type, for increasing value of the next-nearest-neighbor (NNN) hopping. According to Pereira et al. (Refs. [11,12]), for the $B=0$ case, there are vacancy localized states even when $t^{\prime} \neq 0$. Although the NNN hopping breaks the electron-hole symmetry, the localized states are still preserved. Here, we study the nonzero magnetic field case, and we still observe localization peaks. Breaking of the e-h symmetry leads to a displacement of the two peaks, and this displacement (as we show in Fig. 13) depends linearly on the NNN hopping energy $t^{\prime}$. One of the peaks moves toward the $n=-1$ Landau level, whereas the other moves to the zeroth Landau level. Although the two new peaks are clearly visible in the bend resistance, they are not so distinguishable in the DOS. The DOS exhibits considerable broadening, and the two peaks are barely visible after temperature smoothing. A closer look in the LDOS for $t^{\prime} \neq 0$ (not shown) reveals a strong localization on the horizontal, zigzag edges. This edge localization causes this wide background in DOS and masks the narrow vacancy localization peaks.

The linear energy dependence of the new peaks can be further explained if compared with the zero field results of Ref. [11]. The introduction of a nonzero NNN hopping shifts the whole Landau spectrum by $\Delta E=3\left|t^{\prime}\right|$. According to 


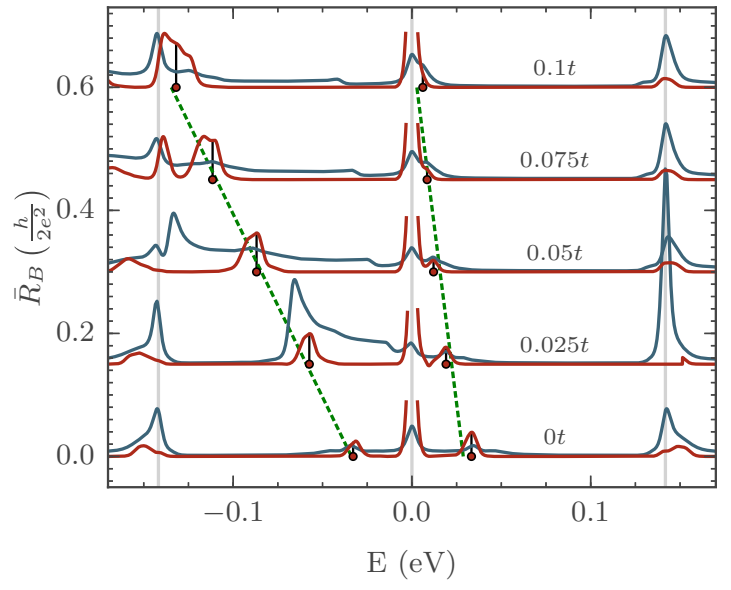

FIG. 13. Smoothed and averaged bend resistances $\left(\bar{R}_{B}\right.$; red curves), and total DOS (blue curves) for increasing value of the next-nearest-neighbor hopping energy $t^{\prime}$. All results are obtained for the SV disorder type $(n=0.01 \%)$, for $N=10$ different disorder distributions. Magnetic field is $B=20 \mathrm{~T}$, and $T=16 \mathrm{~K}$. Green, dashed lines are linear fits of the $\bar{R}_{B}$ peak energy versus the NNN hopping energy $t^{\prime}$. Results for $t^{\prime} \neq 0$ are displaced horizontally by $\Delta E=-3\left|t^{\prime}\right|$ in order to align the Landau levels. The $R_{B}$ peaks at the zeroth Landau level are cut off above $0.09 h /\left(2 e^{2}\right)$ for clarity.

Ref. [11], the shift of the zero mode is less than $\Delta E$, and proportional to $t^{\prime}$. If we assume that the two localized states that we obtain originate from this shifted zero mode, than we expect them to also shift linearly and follow the zero mode. On the other hand, the two linear coefficients in Fig. 13 are different (the distance between the peaks increases with $t^{\prime}$ ). If we assume that the parabolic $E(B)$ dependence is preserved, then we can conclude that $t^{\prime}$ also modifies the scaling coefficient $\alpha$, which is then a linear function of $t^{\prime}$.

\section{CONCLUSIONS}

To conclude, we studied electron transport in graphene Hall bars in quantizing magnetic fields in the presence of three different types of vacancy disorder. All three types of vacancy disorder induce new states in the relativistic Landau spectrum, but these states behave differently depending on the disorder type. The new states, localized around monovacancies, are indirectly observable in the bend resistance and in the total DOS, but only for vacancy concentrations below a critical concentration. These states are localized mostly only on one sublattice, but at the localization energy they spread on both sublattices in $C_{3 v}$-symmetric, flower-like patterns. Another interesting feature is the different behavior of the two monovacancy distribution types. SVA disorder, although inducing an approximately equal number of states as SV disorder (compare DOSs in Fig. 4 for these two disorder types for $n=0.01 \%$ ), creates considerably different results in the bend resistance. The origin of these differences is not known, and requires further study. We speculate that these differences might come from different current flow patterns around different types of vacancy pairs. For example, Ref. [25] showed that vacancy coupling does not depend on their type. However, we showed that these states have a certain symmetry; therefore the coupling strength will also depend on direction, and not only on distance. Divacancies also cause localization, but for fields that we consider, their localization energies are much closer to those of the relativistic LLs, which makes them harder to observe experimentally. Since they do not break the sublattice symmetry, they are usually $C_{2 v}$ symmetric, and they have a constant localization length.

Depending on the ratio between the average vacancyvacancy distance (which depends on the vacancy concentration) and the field strength, localized states around several monovacancies can bond together, forming localized bond states. These bond states have a localization energy different from that of an isolated monovacancy, but on average they spread equally around this energy. The localization energy around a single monovacancy is proportional to the square root of the magnetic field, while the localization radius (and consequently the possible radius of the bond states) scales with the cyclotron radius. The behavior of divacancies is different. Their localization energy scales linearly with the field, and their localization length is independent of the field. Based on this, whether they form bond states depends solely on their mutual distance, and not on the field strength.

A decomposition of the bend resistance reveals that only one transmission function $\left(T_{41}\right)$ is responsible for the appearance of additional peaks in $R_{B}$, which we additionally connect with the vacancy-guided current flow inside the system. All these results are slightly modified when a nextnearest-neighbor interaction is included, and the symmetry between electrons and holes is broken.

\section{ACKNOWLEDGMENT}

This work was supported by the Methusalem program of the Flemish government.
[1] K. S. Novoselov, A. K. Geim, S. V. Morozov, D. Jiang, Y. Zhang, S. V. Dubonos, I. V. Grigorieva, and A. A. Firsov, Science 306, 666 (2004).

[2] Y. Zhang, Y. W. Tan, H. L. Stormer, and P. Kim, Nature (London) 438, 201 (2005).

[3] K. S. Novoselov, A. K. Geim, S. V. Morozov, D. Jiang, M. I. Katsnelson, I. V. Grigorieva, S. V. Dubonos, and A. A. Firsov, Nature (London) 438, 197 (2005).
[4] Y. Zhang, Z. Jiang, J. P. Small, M. S. Purewal, Y.-W. Tan, M. Fazlollahi, J. D. Chudow, J. A. Jaszczak, H. L. Stormer, and P. Kim, Phys. Rev. Lett. 96, 136806 (2006).

[5] G. Li, A. Luican, and E. Y. Andrei, Phys. Rev. Lett. 102, 176804 (2009).

[6] M. M. Ugeda, I. Brihuega, F. Guinea, and J. M. Gómez-Rodríguez, Phys. Rev. Lett. 104, 096804 (2010). 
[7] J. Duffy, J. A. Lawlor, C. Lewenkopf, and M. S. Ferreira, Phys. Rev. B 94, 045417 (2016).

[8] D. A. Ruiz-Tijerina and L. G. G. V. Dias da Silva, Phys. Rev. B 94, 085425 (2016).

[9] M. Droth and G. Burkard, Phys. Rev. B 91, 115439 (2015).

[10] J. Mao, Y. Jiang, D. Moldovan, G. Li, K. Watanabe, T. Taniguchi, M. R. Masir, F. M. Peeters, and E. Y. Andrei, Nat. Phys. 12, 545 (2016).

[11] V. M. Pereira, J. M. B. Lopes dos Santos, and A. H. Castro Neto, Phys. Rev. B 77, 115109 (2008).

[12] V. M. Pereira, F. Guinea, J. M. B. Lopes dos Santos, N. M. R. Peres, and A. H. Castro Neto, Phys. Rev. Lett. 96, 036801 (2006).

[13] A. Cresti, F. Ortmann, T. Louvet, D. Van Tuan, and S. Roche, Phys. Rev. Lett. 110, 196601 (2013).

[14] N. Leconte, F. Ortmann, A. Cresti, and S. Roche, Phys. Rev. B 93, 115404 (2016).
[15] F. Ortmann and S. Roche, Phys. Rev. Lett. 110, 086602 (2013).

[16] J. H. García, L. Covaci, and T. G. Rappoport, Phys. Rev. Lett. 114, 116602 (2015).

[17] C. W. Groth, M. Wimmer, A. R. Akhmerov, and X. Waintal, New J. Phys. 16, 063065 (2014).

[18] H. U. Baranger and A. D. Stone, Phys. Rev. B 40, 8169 (1989).

[19] O. Shevtsov, P. Carmier, C. Petitjean, C. Groth, D. Carpentier, and X. Waintal, Phys. Rev. X 2, 031004 (2012).

[20] M. Büttiker, Phys. Rev. Lett. 57, 1761 (1986).

[21] M. Büttiker, IBM J. Res. Develop. 32, 317 (1988).

[22] D. K. Ferry, S. M. Goodnik, and J. Bird, Transport in Nanostructures (Cambridge University Press, Cambridge, 2009).

[23] S. Datta, Electronic Transport in Mesoscopic Systems (Cambridge University Press, Cambridge, 1995).

[24] M. O. Goerbig, Rev. Mod. Phys. 83, 1193 (2011).

[25] A. L. C. Pereira and P. A. Schulz, Phys. Rev. B 78, 125402 (2008). 